
\title{
26 Research Square \\ GNA14 Stimulation of KLF7 Promotes Malignant Growth of Endometrial Cancer Through Upregulation of HAS2
}

Jing Wang

the First Hospital of Lanzhou University

Fei Teng

the First Hospital of Lanzhou University

Hongxia Chai

the First Hospital of Lanzhou University

Caixia Zhang

the First Hospital of Lanzhou University

Xiaolei Liang

the First Hospital of Lanzhou University

Yongxiu Yang ( $\nabla$ yongxiuyang@163.com )

The First Hospital of Lanzhou University

Research article

Keywords: endometrial cancer, GNA14, KLF7, HAS2, cancer development

Posted Date: November 25th, 2020

DOI: https://doi.org/10.21203/rs.3.rs-113265/v1

License: () (1) This work is licensed under a Creative Commons Attribution 4.0 International License.

Read Full License

Version of Record: A version of this preprint was published at BMC Cancer on April 23rd, 2021. See the published version at https://doi.org/10.1186/s12885-021-08202-y. 


\section{Abstract}

Background: Endometrial cancer (UCEC) is one of the most common gynecological malignancies. We previously found that G protein a subunit 14 (GNA14) was highly expressed in UCEC tissues and functioned as oncogene. Krüppel-like factors (KLFs) are transcription factors and play important roles in cancer development. However, the connection between GNA14 and KLF7 in UCEC are unclear. We herein explored the role of GNA14/KLF7 in UCEC development.

Methods: Clinical relevance of GNA14, KLF7 and HAS2 in UCEC was analyzed from TCGA. siRNAs were used to knock down indicated genes. Lentivirus was used to perform overexpression. mRNA and protein expression was detected by qRT-PCR and Western blot. CCK8, colony formation, cell cycle, apoptosis, transwell and wound healing were performed to check cell biology function in vitro. Tumor growth in nude mice was conducted to check in vivo function. RNA sequencing was used to determined dys-regulated genes.

Results: We demonstrated that GNA14 upregulation of KLF7 promoted UCEC carcinogenesis through activation of hyaluronan synthase 2 (HAS2). Firstly, GNA14 promoted the expression KLF7 in UCEC cells. GNA14 was positively correlated with KLF7 in normal and UCEC tissues based on TCGA database. Then, loss-of-function and gain-of-function assays showed that KLF7 promoted cell proliferation, colony formation, cell cycle progression, and migration of UCEC cells. Apoptosis was inhibited by KLF7. KLF7 knockdown also reduced xenografted tumor growth of UCEC cells. Furthermore, RNA sequence results showed that KLF7 regulated thousands of genes, among which HAS2 was downregulated in KLF7 knockdown cells. Based on TCGA database and immunoblotting assays, KLF7 positively regulated HAS2 in UCEC cells and tissues. Lastly, knockdown of HAS2 reversed the oncogenic role of KLF7 on UCEC cell proliferation and migration.

Conclusion: Taken together, we reveal that GNA14/KLF7/HAS2 signaling cascade is a novel contributor to UCEC development.

\section{Background}

Endometrial cancer (UCEC) is one of the commonest malignant tumors in gynecology. During the past decades, the incidence and mortality of UCEC is rose worldwide [1]. Due to the different biological and histopathological characteristics, UCEC can be divided into two subtypes, namely type I and type II endometrial cancer [2,3]. Development of UCEC is driven by various molecular changes, such as inactivation of tumor suppressors or activation of oncogenes. PTEN represents the most frequent inactivated tumor suppressor and PI3KCA is the commonest mutated oncogene in UCEC. Although most of UCEC patients harboring PI3KCA response to PI3K/AKT/mTOR inhibitors treatment [4], some of them relapse and the treatment efficacy is far from satisfactory. Thus, identifying novel UCEC driven molecules may help us develop effective drugs to treat this malignant disease. 
G protein a subunit 14 (GNA14), one of the $\mathrm{G}$ protein a subunits, plays an important role in signaling transduction, such as activation of phospholipase $C$, protein kinase $C$ and MAPK pathway $[5,6]$. Recent studies have shown that GNA14 may play a pivotal role in cancer development. Upregulation of GNA14 participates in the oncogenic function of TNF-a/TNFR1 in gastric tumorigenesis [7]. GNA14 is frequently mutated in anastomosing haemangiomas [8]. Previously, we showed that GNA14 was upregulated in UCEC tissues and knockdown of GNA14 suppressed the proliferation and growth of UCEC cells [9]. GNA14 downregulation also induced UCEC cell apoptosis. However, the downstream molecular events of GNA14 need further investigations in UCEC development.

The Krüppel-like factors (KLFs) family is a type of transcription factor containing a conserved zinc finger domain, which regulates gene transcription activity by binding to the promoter sequence of the target genes [10, 11]. In humans, KLFs consist of 17 members, comprising of KLF1-KLF17. Based on their transcription activity, KLF7 regulates the expression of various genes and participates in cancer development. For example, KLF7 high expression predicts unfavorable prognosis of lung cancer patients. Knockdown of KLF7 inhibits migration and invasion of lung cancer cells [12]. KLF7 is also highly expressed in squamous carcinoma. Elevated KLF7 contributes to the progression of squamous carcinoma [13]. These results suggest that KLF7 is likely an oncogene in different cancers. Nevertheless, the significance of KLF7 and its correlation with GNA14 remain to be determined in UCEC.

In this study, we analyzed the correlation between GNA14 and KLF7 in UCEC based on TCGA database. Then, we explored the role of KLF7 in UCEC by loss-of-function and gain-of-function experiments. Furthermore, we used RNA sequence to investigate the downstream targets of KLF7 in UCEC. We demonstrated that GNA14/KLF7/HAS2 signaling cascade encourages UCEC progression.

\section{Methods}

\section{The Cancer Genome Atlas database}

Correlation between GNA14 and KLF7 was analyzed in normal samples and UCEC samples from The Cancer Genome Atlas (TCGA, http://cancergenome.nih.gov) database.

\section{Cell culture}

Human UCEC cells KLE, Hec-1-A and Hec-1-B cells were obtained from the American Type Culture collection (ATCC). All the cells were maintained in RPMI 1640 (Invitrogen), which was supplied with 10\% fetal bovine serum (FBS, Gibco) and $1 \%$ antibiotics solution (Corning), at $37^{\circ} \mathrm{C}$ with $5 \% \mathrm{CO}_{2}$.

\section{GNA14, KLF7 and HAS2 interference}

siRNA was used to interfere GNA14, KLF7 and HAS2 in UCEC cells. siRNAs against negative control, GNA14, KLF7 and HAS2 were purchased from GenePharma and were transfected into KLE, Hec-1-A and Hec-1-B cells by RNAiMAX (Invitrogen). 48 hours later, the cells were subjected to immunoblotting 
analysis of knockdown efficiency and functional experiments. For in vivo experiments, lentivirusmediated KLF7 knockdown was performed to establish stable cell lines. siRNA sequences were as follow: siGNA14, 5'-CTACAGATACAGACAATAT-3'; siKLF7-1: 5'-CCGGCUACUUCUCAGCUUU-3'; siKLF7-2; 5'GGUGAGGACUUGGACUGUU-3', and siHAS2; 5'-CCAGUAUCAGUUUGGUUUA-3'.

\section{GNA14 and KLF7 overexpression}

Lentivirus was used to overexpress GNA14 and KLF7 in UCEC cells. The coding sequence of GNA14 or KLF7 was cloned into PCDH vectors. Lentivirus was packaged in 293FT cells and were concentrated using PEG6000. The virus was harvested to infect UCEC cells and the overexpression efficiency was detected by immunoblotting. Then the cells were subjected to functional experiments.

\section{Immunoblotting}

Total proteins were extracted from cells using RIPA buffer (Beyotime, Shanghai, China), containing protease inhibitor cocktail and phosphatase inhibitor cocktail (Roche). Protein concentration was detected by BCA kit (Beyotime). Subsequently, equal amount of total proteins was separated on 10-15\% SDS-PAGE gels and transferred onto PDVF membranes. After incubating with $5 \%$ skim milk, primary, and secondary antibodies, the protein abundance was measured on a chemiluminescence detector. Primary antibody against GNA14 was from Abnova (Taiwan). HAS2 antibody was from Invitrogen. KLF7 and GAPDH antibody, and all the secondary antibodies were from SantaCruz.

\section{Quantitative Real Time PCR (qRT-PCR)}

Total RNA was extracted from UCEC cells using Trizol regent, following to the manufacturer's instructions. RNA was reversely transcribed using M-MLV reverse transcriptase (Promega). The abundance of cDNA was detected by SYBR master mixture. Primer sequences were as follow: HAS forward, 5'TCCTGGATCTCATTCCTCAGC-3' , and reverse, 5'-TGCACTGAACACACCCAAAATA-3'; GAPDH forward, 5'TGACTTCAACAGCGACACCCA -3', and reverse, 5'-CACCCTGTTGCTGTAGCCAAA-3'.

\section{Cell proliferation}

Cell proliferation was detected by CCK8 assay. A total of 2000 UCEC cells were in triplicate seeded into 96-well plates, which contained 100 ul culture medium in each well. At indicated time, 10 ul of CCK8 regent was added into each well and the plates were shocked for 30 seconds, followed by incubation at $37^{\circ} \mathrm{C}$ for 3 hours. Then OD value at $450 \mathrm{~nm}$ was measured on the microplate. Cell viability was normalized to the OD450 of day 1 (OD450 value of the cells 8 hours after seeding).

\section{Colony growth}

UCEC cells were seeded into 6-well plates at equal concentration. 8-10 days later, colonies were formed and washed by PBS for three times. Then, they were fixed by methyl alcohol and stained by crystal violet. Lastly, the colonies were washed by clear water and dried at room temperature. 


\section{Cell cycle}

Cell cycle was detected by PI staining and was analyzed on flow cytometry. Indicated cells were seeded in triplicate in 6-well plates. The cells were harvested and washed by PBS for two times. Then the cells were stained by $\mathrm{Pl}$ and cell cycle was analyzed on flow cytometry.

\section{Apoptosis}

Apoptosis was detected by $\mathrm{PI} /$ Annexin $\mathrm{V}$ staining and was analyzed on flow cytometry. UCEC cells were seeded in triplicate in 6-well plates. The cells were trypsinized by EDTA-free trypsin and washed by PBS for two times. Then the cells were stained by PI/Annexin $\mathrm{V}$ and apoptosis was analyzed on flow cytometry.

\section{In vivo tumorigenesis}

6-week-old immunodeficient nude mice (female, BALB/c) were obtained from Charles River (Beijing, China). Equal number of shCtrl and shKLF7 Hec-1-B cells were subcutaneously implanted into the mice, which were randomly divided into 5 mice per group. Tumors were formed and the mice were euthanatized. Mice were sacrificed by using carbon dioxide euthanasia method according to the protocols. Then tumors were collected for photographing and tumor weighting. All animal experiments were compliant with ethic regulations and approved by the First Hospital of Lanzhou University. The experiments were carried out according to to Institutional Animal Care and Use Committee guidelines of the First Hospital of Lanzhou University.

\section{Transwell}

Migration was assessed by transwell assay. Equal amount of siCtrl, siKLF7-1 and siKLF7-2 Hec-1-B cells in 200 ul FBS-free culture medium were seeded onto the upper layer of transwell chamber. The lower chamber contained 500 ul 10\% FBS culture medium. 24 hours later, the cells on the upper layer were removed and the cells on the lower layer were fixed by methyl alcohol and stained by crystal violet. Migrated cells were photographed under the microscope.

\section{Wound healing}

Migration was assessed by wound healing assay. The cells were seeded into 6-well plates. After reaching $90 \%$ confluence, 200 ul pipettes were used to create wounds with similar breadth in each well. Cell supernatants were removed and fresh culture medium was added into each well. Cells were photographed at 0 hour and 48 hours later under microscope.

\section{Statistical analysis}

Statistical difference was analyzed by GraphPad prism software. Student's t test was applied to compare the difference between two groups and one-way ANOVA analysis was used to analyze the difference among multiple groups. Statistical significance was considered when $p<0.05$. 


\section{Results}

\section{GNA14 expression is correlated with KLF7 expression in UCEC.}

We previously found that GNA14 is an oncogene in UCEC. To explore the downstream mechanisms of GNA14 in UCEC, we firstly analyzed the positively correlated genes of GNA14 from TCGA database. We observed that there was a positive relationship between GNA14 and KLF7 mRNA abundance in both normal and UCEC tissues (Fig. 1A and 1B). We therefore investigated whether GNA14 regulated KLF7 in UCEC cells by knocking down and overexpressing GNA14. Immunoblotting results showed that GNA14 knock down reduced the expression of KLF7, whereas GNA14 overexpression promoted the expression KLF7 in UCEC cells (Fig. 1C). Taken together, GNA14 positively regulated KLF7 in UCEC cells and tissues.

\section{KLF7 promotes the proliferation and migration of UCEC cells.}

Since GAN14 is an oncogene and is positively correlated with KLF7 in UCEC, we next intend to explore the function of KLF7 in UCEC. KLF7 was knocked down using siRNA in UCEC cells and the cells were subjected to cell proliferation and migration experiments. Immunoblotting results showed that KLF7 was efficiently silenced in siKLF7-1 and siKLF7-2 cells (Fig. 2A). Interference of KLF7 significantly suppressed the proliferation of KLE and Hec-1-A cells (Fig. 2B and 2C). In addition, KLF7 knockdown also led to reduced colony growth in KLE, Hec-1-A and Hec-1-B cells (Fig. 2D-2F). To address whether KLF7 is sufficient to induce the phenotypes, we overexpressed KLF7 in KLE cells (Fig. 2G). We found that KLF7 ectopic expression enhanced the proliferation and colony formation ability of KLE cells (Fig. 2H and 2I). These results suggest that KLF7 acts as an oncogene in UCEC.

\section{KLF7 promotes cell cycle arrest and induces apoptosis of UCEC cells}

To study the effect of KLF7 on cell cycle progression and apoptosis, we subjected the cells to PI staining and Pl/Annexin V staining. We found that KLF7 knockdown led to enhanced apoptosis in Hec-1-A and Hec-1-B cells (Fig. 3A-3C). PI staining results showed that KLF7 knockdown led to cell cycle arrest at G0/G1 phase in Hec-1-A and Hec-1-B cells (Fig. 3D-3G). By contrast, KLF7 ectopic expression resulted in decreased apoptosis and reduced cell number of G0/G1 phase in Hec-1-A and Hec-1-B cells (Fig. 3H-3K). Taken together, KLF7 promotes cell proliferation by regulating apoptosis and cell cycle progression.

\section{KLF7 promotes UCEC cell migration.}

Next, we assessed the role of KLF7 on UCEC cell migration by using wound healing and transwell assay. Would healing results showed that knockdown of KLF7 significantly suppressed the migration of Hec-1-A, Hec-1-B and KLE cells (Fig. 4A-4F). Moreover, the migration capacity of Hec-1-B cells was reduced after KLF7 knockdown by migration assay (Fig. 4G and 4H). Furthermore, KLF7 overexpression enhanced the migration of KLE cells (Fig. $4 \mathrm{I}$ and $4 \mathrm{~J}$ ). Above results indicate that KLF7 expression is critical for UCEC cell migration. Thus, KLF7 is critical for UCEC cell migration. 
Next, we explored the in vivo role of KLF7 in UCEC. Hec-1-B cells expressing shCtrl and shKLF7 lentivirus were subcutaneously injected into the immunodeficient nude mice (6-week-old, male BALB/c) to develop xenografic tumor models. Tumor growth was evaluated and tumor weight was assessed after sacrifice. We found that KLF7 knockdown suppressed the growth of Hec-1-B cells in nude mice (Fig. 5A and 5B). These results suggest that KLF7 contributes to UCEC carcinogenesis.

\section{KLF7 knockdown results in dysregulation of numerous genes.}

To profile the downstream effectors of KLF7 in UCEC cells, we subjected siCtrl and siKLF7 UCEC cells to RNA sequence analysis. Differentially expressed genes were identified following the criterion: fold change $>1.5$ and $p<0.05$. We showed that a total of 2464 genes were upregulated and a total of 2377 genes were downregulated after KLF7 knockdown (Fig. 6A). GSEA analysis showed that TNFa signaling via NF-kB was suppressed, while oxidative phosphorylation and hypoxia were activated after KLF7 knockdown (Fig. 6B). We also found that HAS2 was downregulated in siKLF7 cells based on RNA sequence data. qRT-PCR showed that HAS2 expression was decreased and increased in KLF7 knockdown and overexpressed cells (Fig. 6C). There was a positive correlation between KLF7 expression and HAS2 expression in UCEC tissues, which was analyzed from TCGA database (Fig. 6D). Taken together, KLF7 upregulates HAS2 in UCEC cells and tissues.

\section{Knockdown of HAS2 restores UCEC cell growth.}

RNA sequence and qRT-PCR results showed that KLF7 upregulated HAS2, but whether HAS2 contributes to the oncogenic function of KLF7 in UCEC should be determined. To address this question, we silenced HAS2 in KLF7 overexpressed KLE cells. Immunoblotting results showed that KLF7 overexpression upregulated HAS2 in KLE cells, and HAS2 knockdown efficiently downregulated HAS2 in KLE cells (Fig. 7A). CCK8 and colony formation results demonstrated that HAS2 silencing suppressed the cell proliferation and colony formation in KLF7 overexpressed KLE cells (Fig. 7B and 7C). Furthermore, HAS2 knockdown inhibited migration in KLF7 overexpressed KLE cells (Fig. 7D). Therefore, HAS2 upregulation by KLF7 promotes UCEC cell proliferation and migration.

\section{Discussion}

Endometrial cancer (UCEC) is one of the most common gynecological malignancies, accounting for 20$30 \%$ of all female reproductive system cancers. In recent years, scientists have made many efforts to analyze the mechanism of the occurrence and development of endometrial cancer. The progression of UCEC is closely related to the abnormalities of KRAS, PTEN and $\beta$-catenin [14-16]. However, although the above genes play an important role in the occurrence and development of endometrial cancer, it is still unable to fully analyze the tumorigenesis and development of all endometrial cancer patients. The pathogenic mechanism of endometrial cancer still needs to be further explored.

We previously found that GNA14 was highly expressed in UCEC patients. Knockdown of GNA14 blocked cell growth and induced apoptosis in UCEC cells [9]. These results suggest that GNA14 is likely a 
promising drug target in UCEC. However, the downstream molecular mechanisms should be investigated. In this study, we found that KLF7 was positively regulated by GNA14 in UCEC cells. Interestingly, there was a positive correlation between GNA14 and KLF7 in UCEC patients. Thus, KLF7 might be the downstream effector of GNA14 in UCEC. As a transcription factor, KLF7 participates in the development of various cancers. For instance, KLF7 is negatively regulated by P53 pathway and upregulation of KLF7 promotes the development of pancreatic ductal adenocarcinoma by regulating golgi complex integrity [17]. KLF7 is also overexpressed in gastric cancer samples and it encourages the migration and invasion of gastric cancer cells [18]. Furthermore, KLF7 can also be targeted by microRNA, such as miR-185 and miR-193a, in lung cancer $[19,20]$. Overall, KLF7 functions as on oncogene in different cancers and its upregulation can be regulated by various factors. Based on the results as demonstrated by other studies, we predicted that GNA14 upregulation of KLF7 might partly contribute to UCEC development. As expected, loss-of-function and gain-of-function experiments demonstrated that KLF7 encouraged cell cycle progression, proliferation, colony growth, migration, and tumorigenesis of UCEC cells. Apoptosis was inhibited by KLF7 in UCEC cells. Therefore, we concluded that KLF7 contributed to the oncogenic role of GNA14 in UCEC.

Because KLF7 is a transcription factor, there may be a downstream effector of KLF7 in UCEC. Based on our RNA sequence and validation data, we showed that KLF7 positively regulated the expression of hyaluronan synthase 2 (HAS2), which is a critical enzyme controlling the biosynthesis of hyaluronan. A study showed that HAS2 regulation of hyaluronan synthesis is important for liver fibrosis development [21]. Recently, a large numbers of studies have reported the function and significance of HAS2 in cancer development. HAS2 increases breast cancer cell invasion through inhibiting tissue metalloproteinase inhibitor 1 (TIMP-1) [22]. Silencing of HAS2 promotes the radiosensitivity of cancer cells [23]. Overexpression of HAS2 is correlated with malignant function of breast cancer cells [24]. Although hyaluronan synthase and its product hyaluronan are likely related to the progression of UCEC [25-27], there are no direct evidences which demonstrate the oncogenic role of HAS in UCEC. Furthermore, the upstream regulators of HAS2 in UCEC are underdetermined. We here found that KLF7 served as a positive regulator of HAS2 in UCEC cells. TCGA database showed a positive correlation between KLF7 and HAS2. We also demonstrated that HAS2 knockdown reversed the phenotypes observed in KLF7 overexpressed UCEC cells. Taken together, KLF7 upregulation of HAS2 promotes UCEC proliferation.

\section{Conclusions}

In summary, we provided the evidence that GNA14/KLF7/HAS2 signaling cascade encouraged the development of UCEC. This signaling cascade was also a promising diagnostic candidate for UCEC. Blocking this cascade may benefit UCEC patients.

\section{Abbreviations}

UCEC, Endometrial cancer; GNA14, G protein a subunit 14; KLFs, Krüppel-like factors; HAS2, hyaluronan synthase 2; ATCC, American Type Culture collection; FBS, fetal bovine serum; qRT-PCR, Quantitative Real 
Time PCR; TIMP-1, inhibiting tissue metalloproteinase inhibitor 1.

\section{Declarations}

\section{Ethics approval and consent to participate}

Animal experiments were approved by the First Hospital of Lanzhou University, Key Laboratory for Gynecologic Oncology Gansu Province.

\section{Consent for publication}

Not Applicable.

\section{Availability of data and material}

All data generated or analyzed during this study are included in this published article.

\section{Acknowledgements}

Not Applicable.

\section{Funding}

The research was financially supported by National Natural Science Foundation of China (No. 81960278 and No. 81601351) and The Fundamental Research Funds for the Central Universities (No: Izujbky-2020kb28). Funding bodies did not have any role in the design of the study, data analysis or writing the manuscript.

\section{Authors' contributions}

Y.Y. and X.L. designed the study and wrote the manuscript. J.W. performed the most of experiments. F.T. and H.C. performed in vivo tumorigenesis assay. C.Z. analyzed the data. All authors have read and approved the manuscript

\section{Competing interests}

These authors declare no conflicts of interest.

\section{References}

1. Braun M, Overbeek-Wager E, Grumbo R. Diagnosis and Management of Endometrial Cancer. Am Fam Physician. 2016;93(6):468-74.

2. Shang Y. Molecular mechanisms of oestrogen and SERMs in endometrial carcinogenesis. Nat Rev Cancer. 2006;6(5):360-8. 
3. Amant F, Moerman P, Neven P, Timmerman D, Van Limbergen E, Vergote I. Endometrial cancer. Lancet. 2005;366(9484):491-505.

4. Janku F, Wheler J, Westin S, Moulder S, Naing A, Tsimberidou A, Fu S, Falchook G, Hong D, GarridoLaguna I, et al. PI3K/AKT/mTOR inhibitors in patients with breast and gynecologic malignancies harboring PIK3CA mutations. Journal of clinical oncology: official journal of the American Society of Clinical Oncology. 2012;30(8):777-82.

5. Neves SR, Ram PT, lyengar R. G protein pathways. Science. 2002;296(5573):1636-9.

6. Lim YH, Bacchiocchi A, Qiu J, Straub R, Bruckner A, Bercovitch L, Narayan D, McNiff J, Ko C, Robinson-Bostom L, et al. GNA14 Somatic Mutation Causes Congenital and Sporadic Vascular Tumors by MAPK Activation. Am J Hum Genet. 2016;99(2):443-50.

7. Oshima H, Ishikawa T, Yoshida GJ, Naoi K, Maeda Y, Naka K, Ju X, Yamada Y, Minamoto T, Mukaida $\mathrm{N}$, et al. TNF-a/TNFR1 signaling promotes gastric tumorigenesis through induction of Noxo1 and Gna14 in tumor cells. Oncogene. 2014;33(29):3820-9.

8. Bean GR, Joseph NM, Folpe AL, Horvai AE, Umetsu SE. Recurrent GNA14 mutations in anastomosing haemangiomas. Histopathology. 2018;73(2):354-7.

9. Wang J, Lv X, Xu F, Wei M, Liu C, Yang Y. GNA14 silencing suppresses the proliferation of endometrial carcinoma cells through inducing apoptosis and G(2)/M cell cycle arrest. Biosci Rep 2018, 38(5).

10. Presnell JS, Schnitzler CE, Browne WE. KLF/SP Transcription Factor Family Evolution: Expansion, Diversification, and Innovation in Eukaryotes. Genome Biol Evol. 2015;7(8):2289-309.

11. Kaczynski J, Cook T, Urrutia R. Sp1- and Krüppel-like transcription factors. Genome Biol. 2003;4(2):206.

12. Niu R, Tang Y, Xi Y, Jiang D. High Expression of Krüppel-like Factor 7 Indicates Unfavorable Clinical Outcomes in Patients with Lung Adenocarcinoma. J Surg Res. 2020;250:216-23.

13. Yang J, Xie K, Wang Z, Li C. Elevated KLF7 levels may serve as a prognosis signature and might contribute to progression of squamous carcinoma. FEBS Open Bio. 2020;10(8):1577-86.

14. Lagarda $H$, Catasus L, Arguelles R, Matias-Guiu X, Prat J. K-ras mutations in endometrial carcinomas with microsatellite instability. J Pathol. 2001;193(2):193-9.

15. Mutter GL, Lin MC, Fitzgerald JT, Kum JB, Baak JP, Lees JA, Weng LP, Eng C. Altered PTEN expression as a diagnostic marker for the earliest endometrial precancers. J Natl Cancer Inst. 2000;92(11):92430 .

16. Machin P, Catasus L, Pons C, Muñoz J, Matias-Guiu X, Prat J. CTNNB1 mutations and beta-catenin expression in endometrial carcinomas. Hum Pathol. 2002;33(2):206-12.

17. Gupta R, Malvi P, Parajuli KR, Janostiak R, Bugide S, Cai G, Zhu LJ, Green MR, Wajapeyee N. KLF7 promotes pancreatic cancer growth and metastasis by up-regulating ISG expression and maintaining Golgi complex integrity. Proc Natl Acad Sci U S A. 2020;117(22):12341-51.

18. Jiang Z, Yu T, Fan Z, Yang H, Lin X. Krüppel-Like Factor 7 is a Marker of Aggressive Gastric Cancer and Poor Prognosis. Cell Physiol Biochem. 2017;43(3):1090-9. 
19. Zhao L, Zhang Y, Liu J, Yin W, Jin D, Wang D, Zhang W: miR-185 Inhibits the Proliferation and Invasion of Non-Small Cell Lung Cancer by Targeting KLF7. Oncol Res 2019, 27(9):1015-1023.

20. An YX, Shang YJ, Xu ZW, Zhang QC, Wang Z, Xuan WX, Zhang XJ. STAT3-induced long noncoding RNA LINC00668 promotes migration and invasion of non-small cell lung cancer via the miR193a/KLF7 axis. Biomed Pharmacother. 2019;116:109023.

21. Yang YM, Noureddin M, Liu C, Ohashi K, Kim SY, Ramnath D, Powell EE, Sweet MJ, Roh YS, Hsin IF, et al: Hyaluronan synthase 2-mediated hyaluronan production mediates Notch1 activation and liver fibrosis. Sci Trans/ Med 2019, 11(496).

22. Bernert $B$, Porsch $H$, Heldin P. Hyaluronan synthase 2 (HAS2) promotes breast cancer cell invasion by suppression of tissue metalloproteinase inhibitor 1 (TIMP-1). J Biol Chem. 2011;286(49):42349-59.

23. Shen YN, Shin HJ, Joo HY, Park ER, Kim SH, Hwang SG, Park SJ, Kim CH, Lee KH. Inhibition of HAS2 induction enhances the radiosensitivity of cancer cells via persistent DNA damage. Biochem Biophys Res Commun. 2014;443(3):796-801.

24. Li P, Xiang T, Li H, Li Q, Yang B, Huang J, Zhang X, Shi Y, Tan J, Ren G. Hyaluronan synthase 2 overexpression is correlated with the tumorigenesis and metastasis of human breast cancer. Int $\mathrm{J}$ Clin Exp Pathol. 2015;8(10):12101-14.

25. Nykopp TK, Rilla K, Tammi MI, Tammi RH, Sironen R, Hämäläinen K, Kosma VM, Heinonen S, Anttila M. Hyaluronan synthases (HAS1-3) and hyaluronidases (HYAL1-2) in the accumulation of hyaluronan in endometrioid endometrial carcinoma. BMC Cancer. 2010;10:512.

26. Yabushita H, Kishida T, Fusano K, Kanyama K, Zhuo L, Itano N, Kimata K, Noguchi M. Role of hyaluronan and hyaluronan synthase in endometrial cancer. Oncol Rep. 2005;13(6):1101-5.

27. Paiva P, Van Damme MP, Tellbach M, Jones RL, Jobling T, Salamonsen LA. Expression patterns of hyaluronan, hyaluronan synthases and hyaluronidases indicate a role for hyaluronan in the progression of endometrial cancer. Gynecol Oncol. 2005;98(2):193-202.

\section{Figures}

A

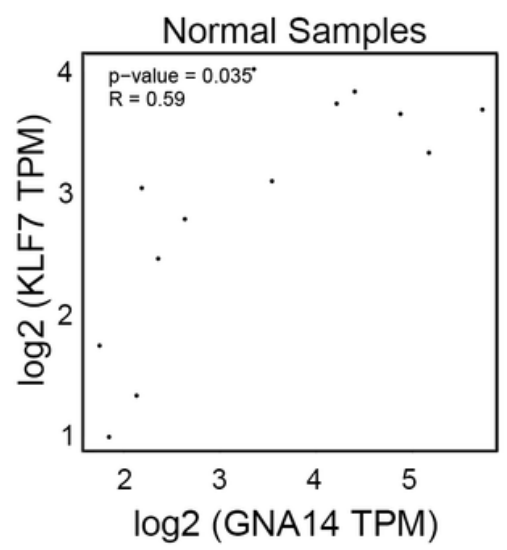

B

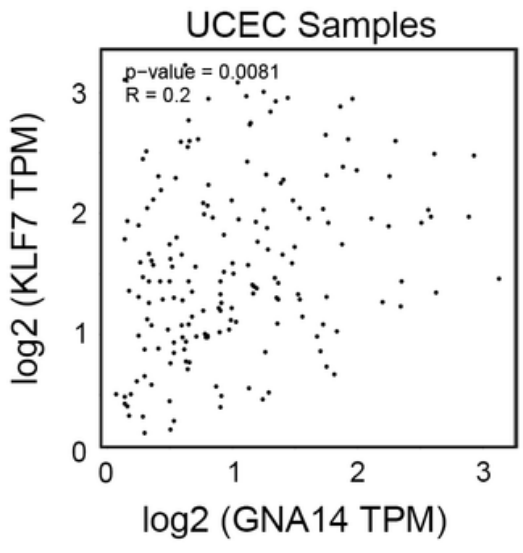

C

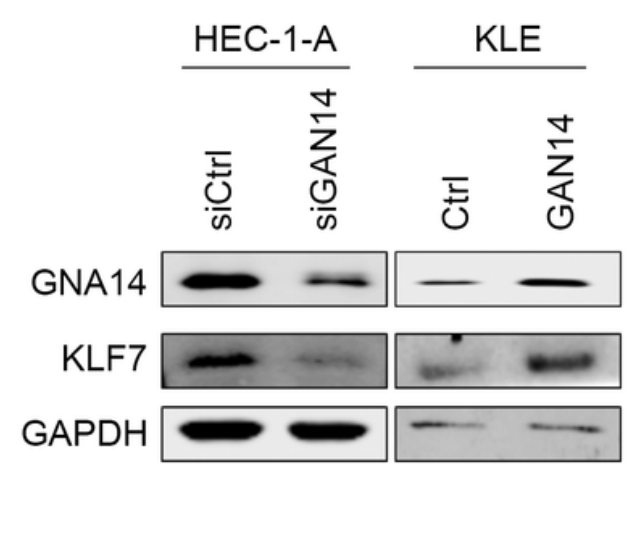


Figure 1

GNA14 upregulates KLF7 in UCEC (A and B) The correlation between GNA14 expression and KLF7 expression was analyzed in normal samples $(A, R=0.59, p=0.035)$ and UCEC samples $(B, R=0.2$, $\mathrm{p}=0.0081$ ) from TCGA database. (C) Immunoblotting analysis of GAN14 and KLF7 in GAN14 knockdown Hec-1-A and GAN14 overexpressed KLE cells. GAPDH acts as internal control.

A

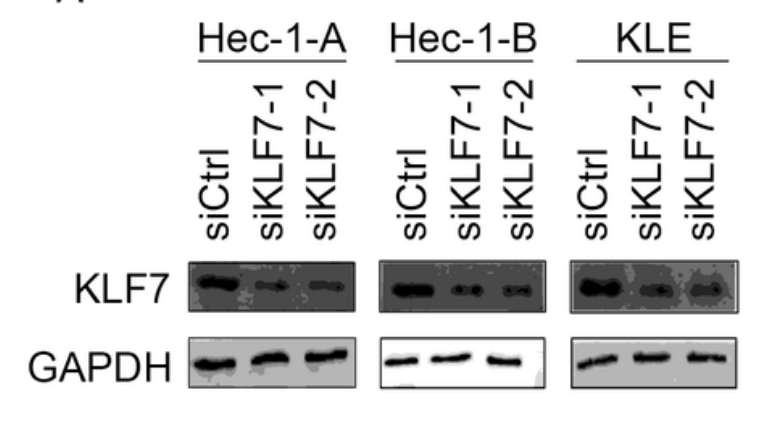

D

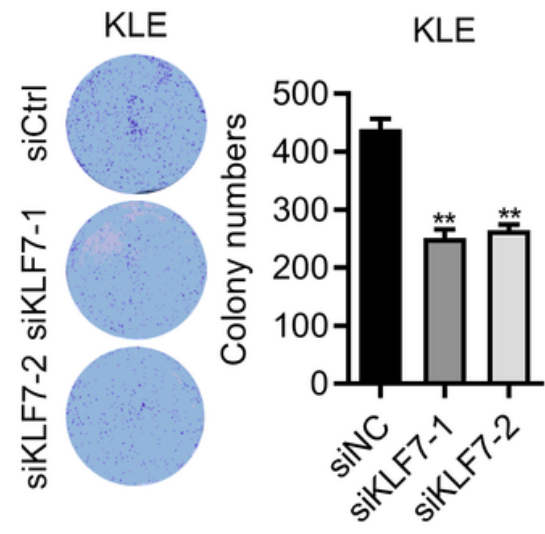

$\mathrm{E}$
B

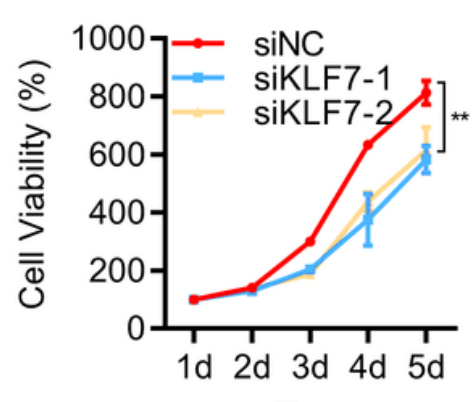

Time
C

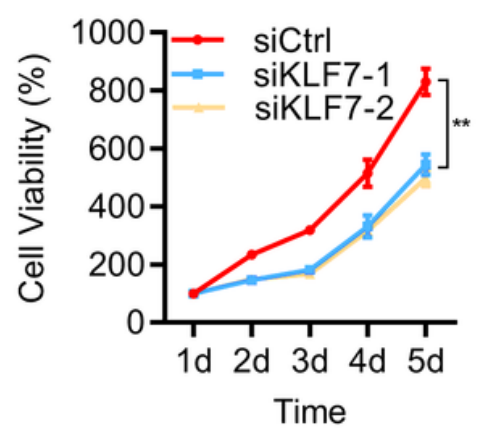

G

$\mathrm{H}$

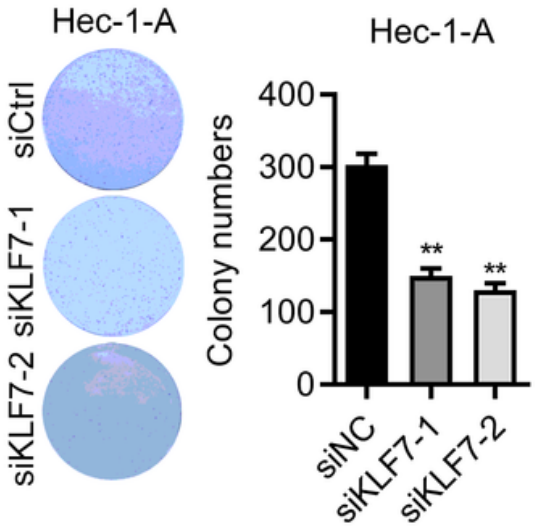

KLE

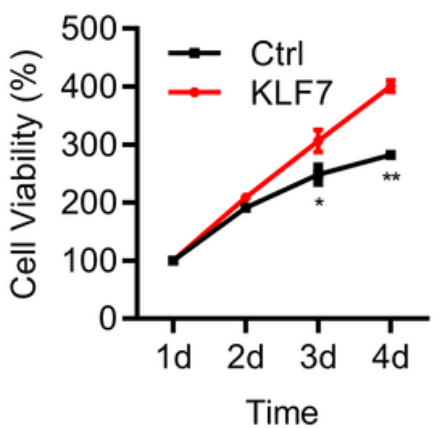

$\mathrm{F}$
Hec-1-B

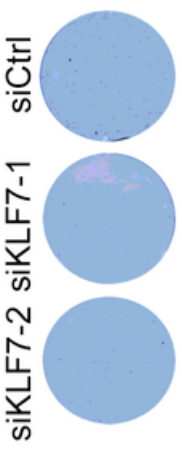

Hec-1-B

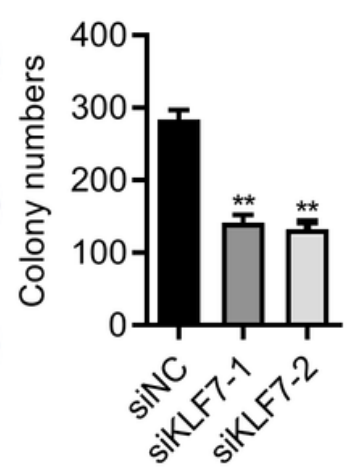

KLE

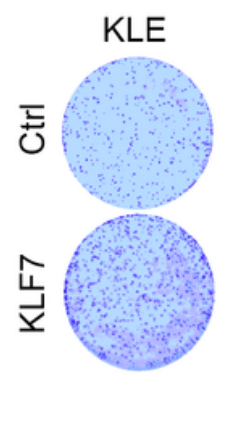

Figure 2

KLF7 promotes UCEC cell proliferation (A) Immunoblotting analysis of KLF7 in siCtrl, siKLF7-1 and siKLF7-2 UCEC cells. (B and C) CCK8 analysis of cell proliferation in siCtrl, siKLF7-1 and siKLF7-2 KLE and Hec-1-A cells. (D-F) Colony formation was assessed in siCtrl, siKLF7-1 and siKLF7-2 KLE, Hec-1-A, 
and Hec-1-B cells. (G) Immunoblotting analysis of KLF7 in Ctrl and KLF7 overexpressed KLE cells. (H) CCK8 analysis of cell proliferation in Ctrl and KLF7 overexpressed KLE cells. (I) Colony formation was assessed in Ctrl and KLF7 overexpressed KLE cells. ${ }^{*} p<0.05 .{ }^{* *} p<0.01 .{ }^{* * *} p<0.001$.

A
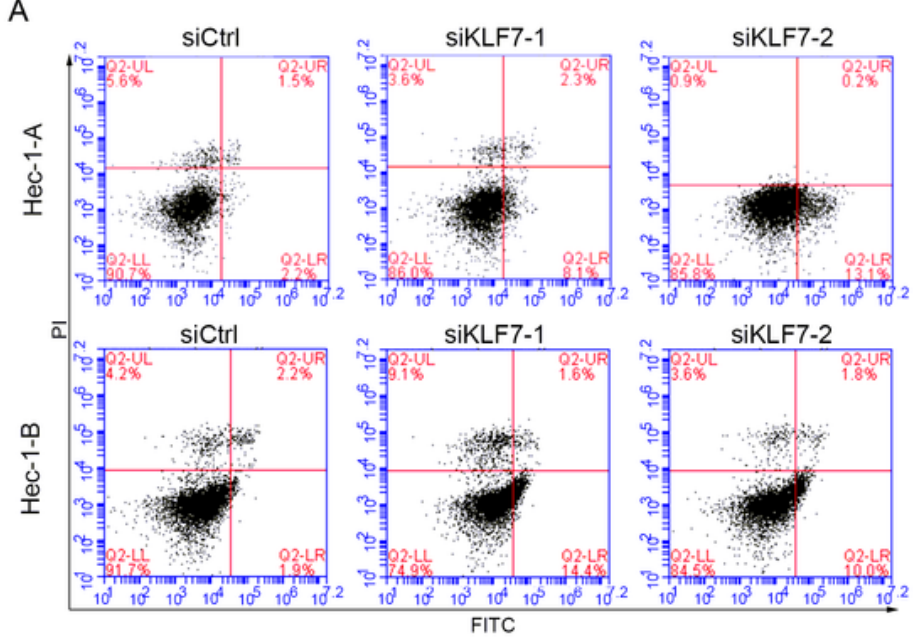

D
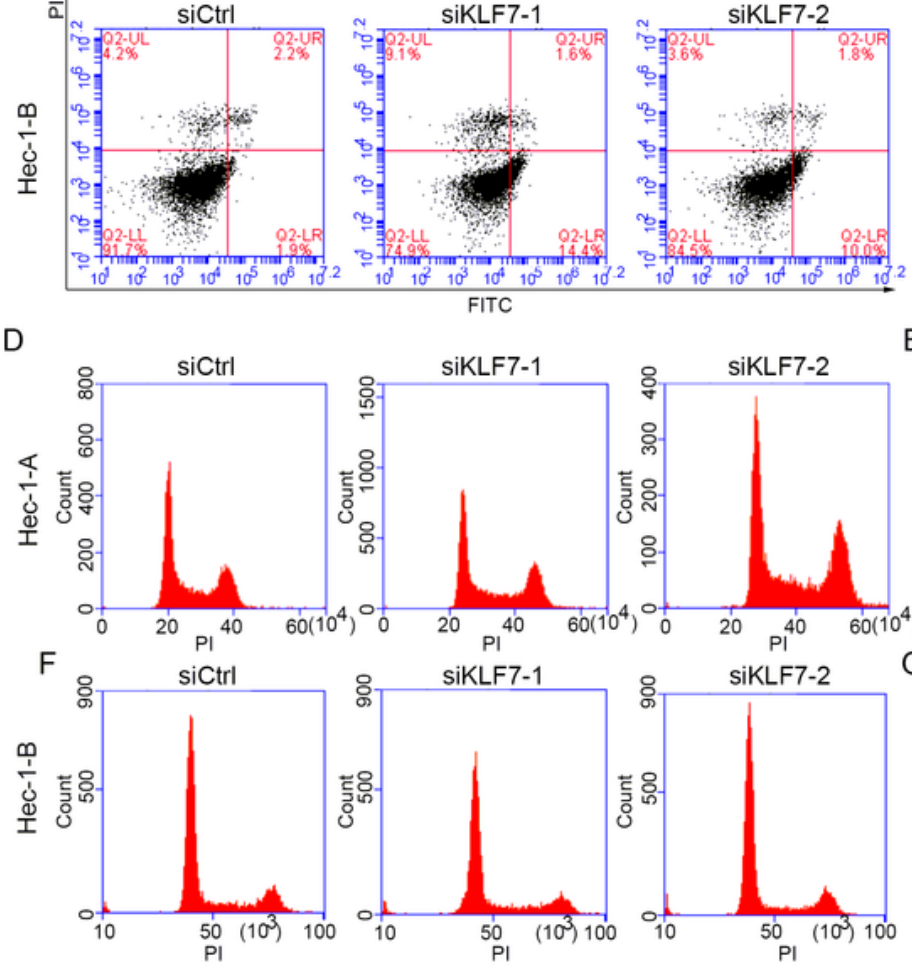

C
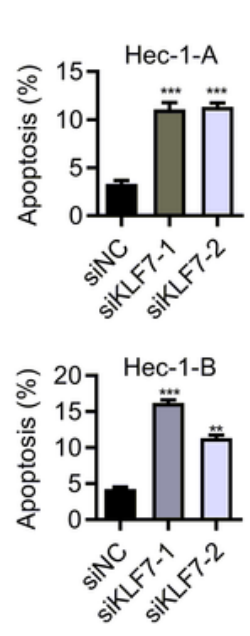

E Hec-1-A
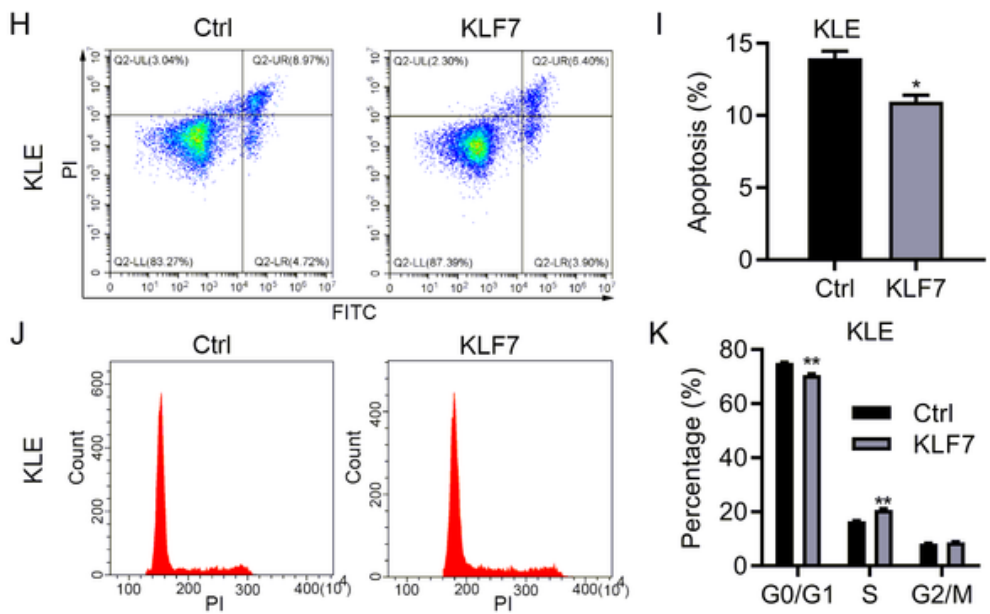

Figure 3

KLF7 regulates cell cycle and apoptosis in UCEC cells (A-C) Apoptosis was examined by $\mathrm{PI} /$ Annexin V staining in siCtrl, siKLF7-1 and siKLF7-2 Hec-1-A and Hec-1-B cells. A, apoptosis results. B and C, 
quantitative results. (D-G) Cell cycle was examined by PI staining in siCtrl, siKLF7-1 and siKLF7-2 Hec-1-A and Hec-1-B cells. D and F, cell cycle results. E and G, quantitative results. ( $\mathrm{H}$ and I) Apoptosis was examined by $\mathrm{Pl} /$ Annexin V staining in Ctrl and KLF7 overexpressed KLE cells. $\mathrm{H}$, apoptosis results. I, quantitative results. ( $\mathrm{J}$ and $\mathrm{K}$ ) Cell cycle was examined by PI staining in Ctrl and KLF7 overexpressed KLE cells. J, cell cycle results. $K$, quantitative results. ${ }^{*} p<0.05 .{ }^{* *} p<0.01$. ${ }^{* \star *} p<0.001$.

A

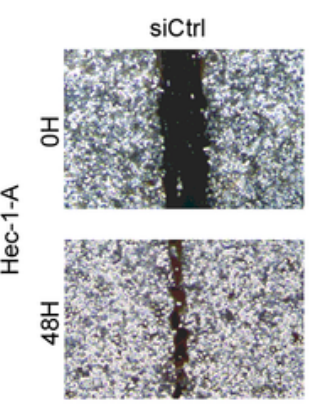

C

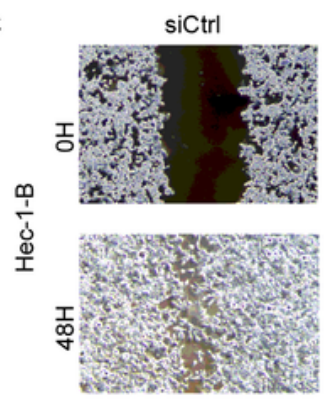

$\mathrm{E}$

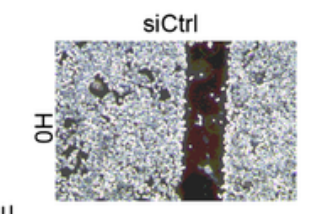

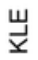

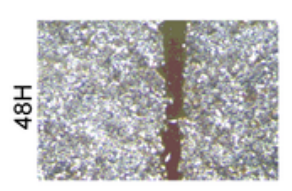

G

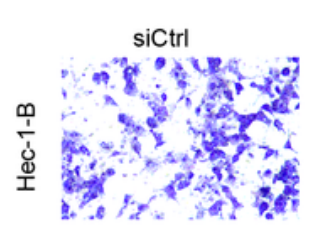

I

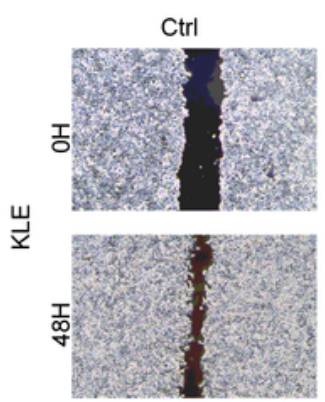

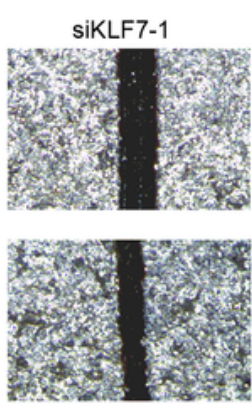

SiKLF7-1

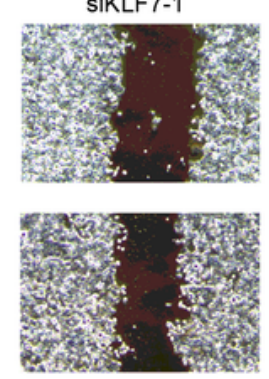

siKLF7-1
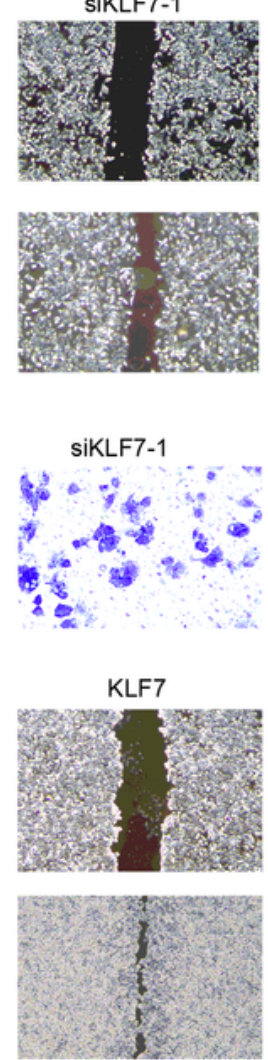

siKLF7-2

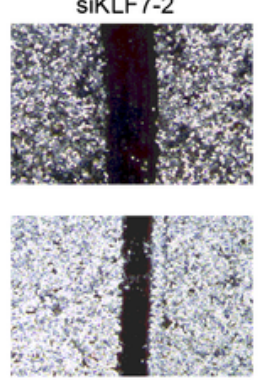

siKLF7-2

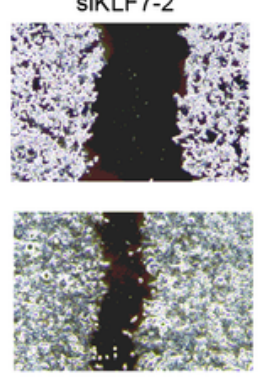

siKLF7-2
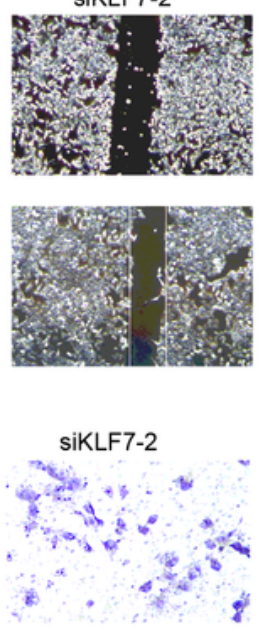

$J$

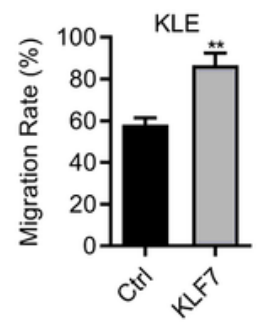

B

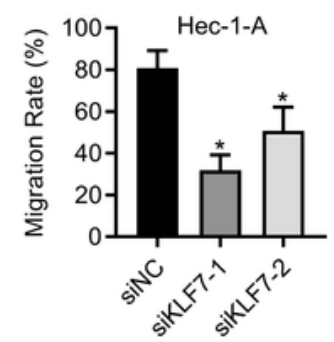

D

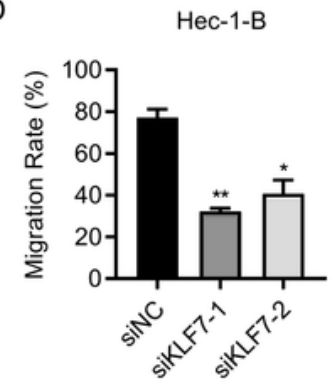

F
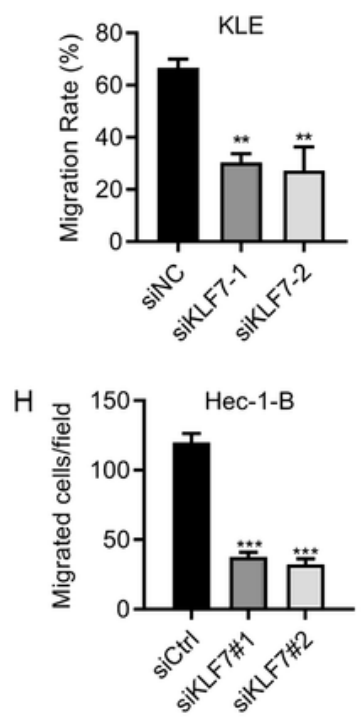

\section{Figure 4}


KLF7 promotes UCEC cell migration (A-F) Migration was determined by wound healing assay in siCtrl, siKLF7-1 and siKLF7-2 Hec-1-A ( $A$ and B), Hec-1-B ( $C$ and D), and KLE ( $E$ and F) cells. ( $G$ and $H$ ) Transwell detection of migration in siCtrl, siKLF7-1 and siKLF7-2 Hec-1-B cells. (I and J) Migration was determined by wound healing assay in Ctrl and KLF7 overexpressed KLE cells. ${ }^{*} p<0.05 .{ }^{* *} p<0.01 .{ }^{* * *} p<0.001$.

A

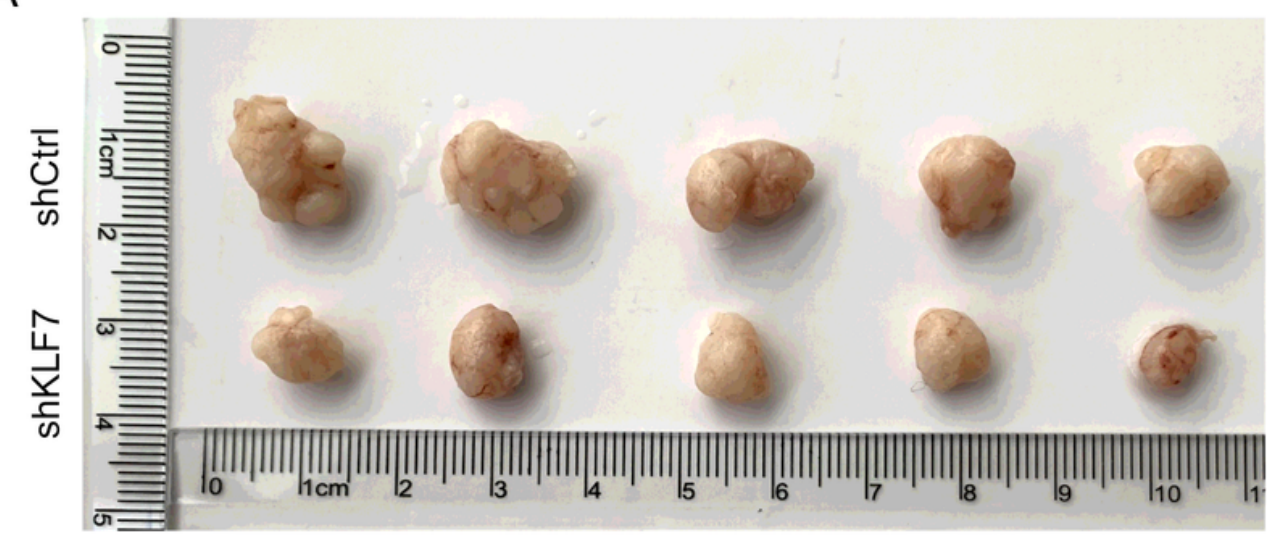

B

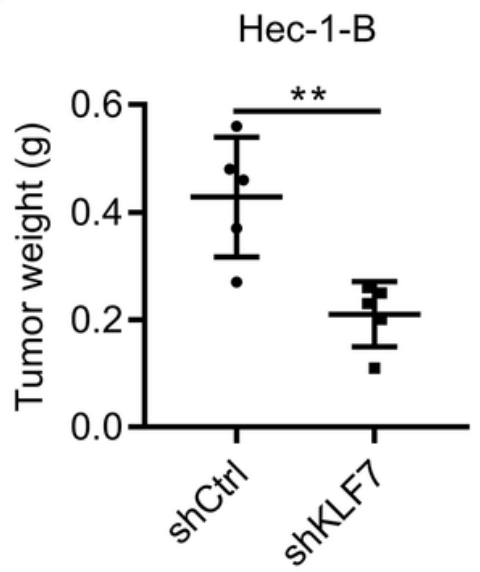

Figure 5

KLF7 knockdown prevents tumorigenesis of UCEC cells Equal number of shCtrl and shKLF7 Hec-1-B cells were subcutaneously implanted into female nude mice. Tumors were photographed after sacrifice. (A) Macroscopic images of tumors derived from shCtrl and shKLF7 Hec-1-B cells in nude mice. (B) Tumor weight was measured. ${ }^{* \star} p<0.01$. 
A
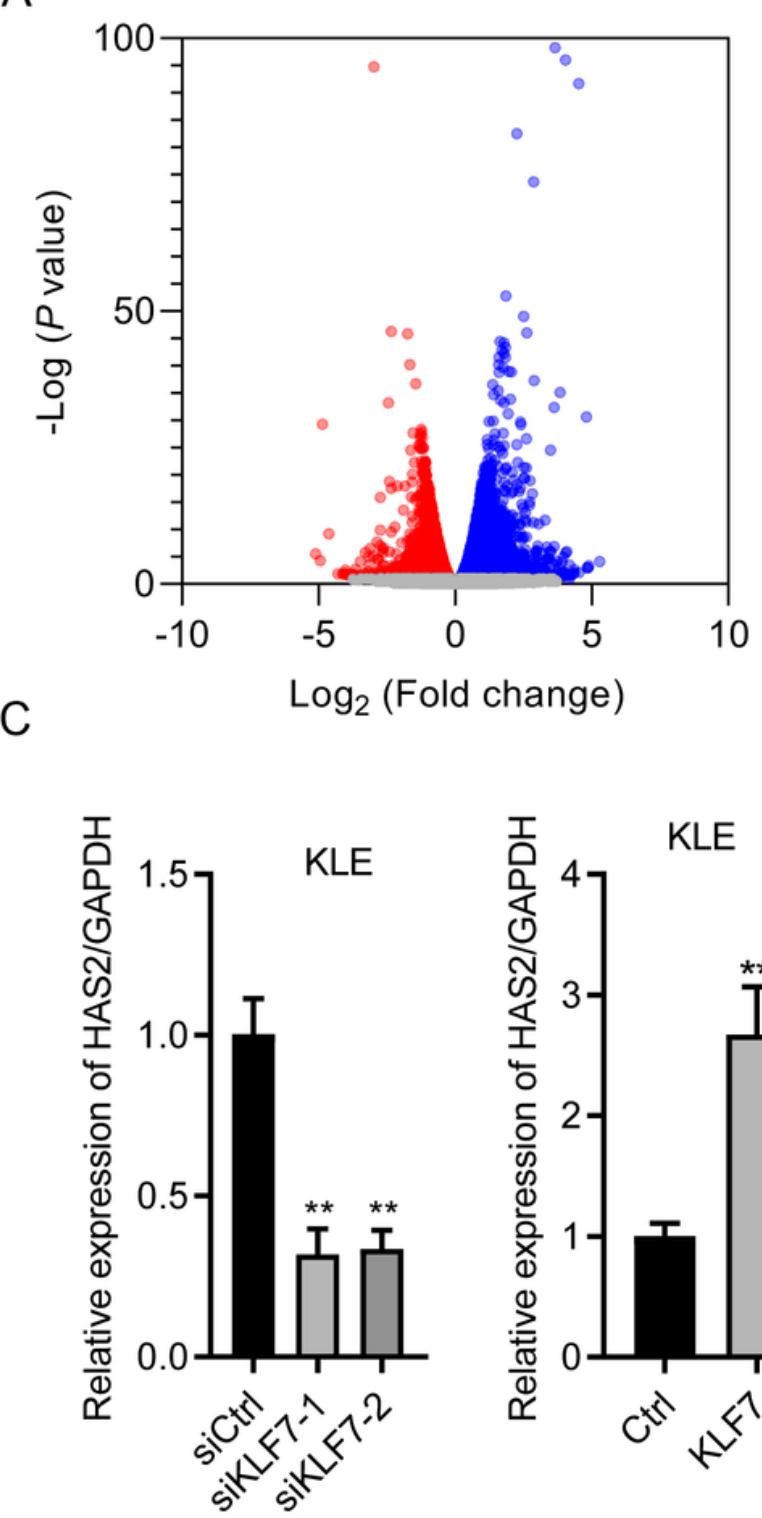

B

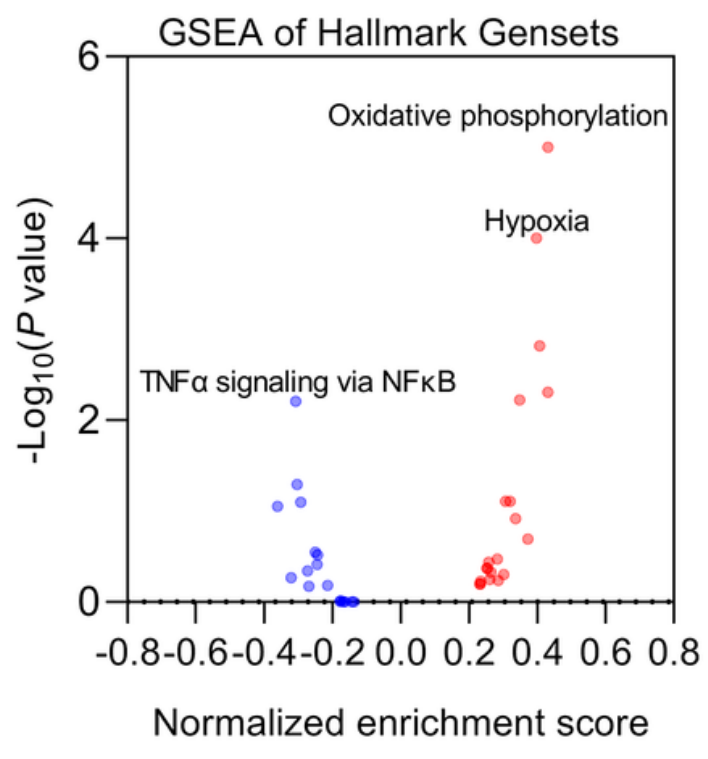

548 UCEC samples from TCGA database

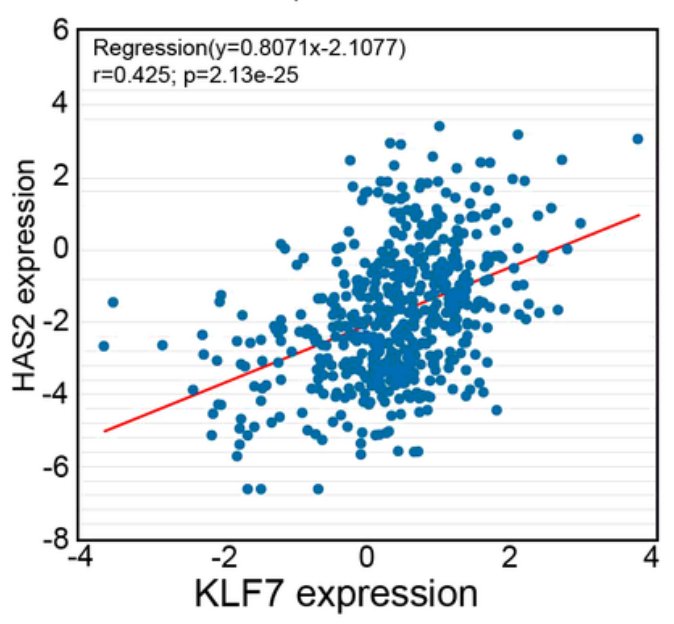

Figure 6

Molecule profiling in UCEC cells after KLF7 knockdown (A) siCtrl and siKLF7 UCEC cells were subjected to RNA sequence. Differentially expressed genes were identified following the criterion: fold change $>1.5$ and $p<0.05$. (A) Upregulated genes were shown as red. Downregulated genes were shown as blue. Gray represented unchanged genes. (B) GESA analysis of signaling pathway after KLF7 knockdown. (C) qRTPCR detection of HAS2 in KLE cells after KLF7 knockdown and overexpression. (D) Correlation between KLF7 expression and HAS2 expression was analyzed in UCEC tissues base on TCGA database. ${ }^{* *} \mathrm{p}<0.01$. 
A

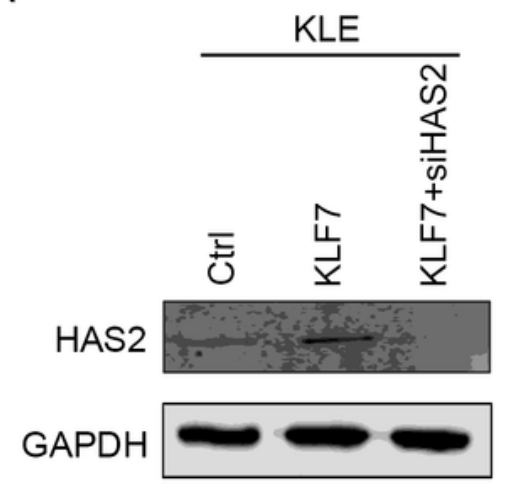

C

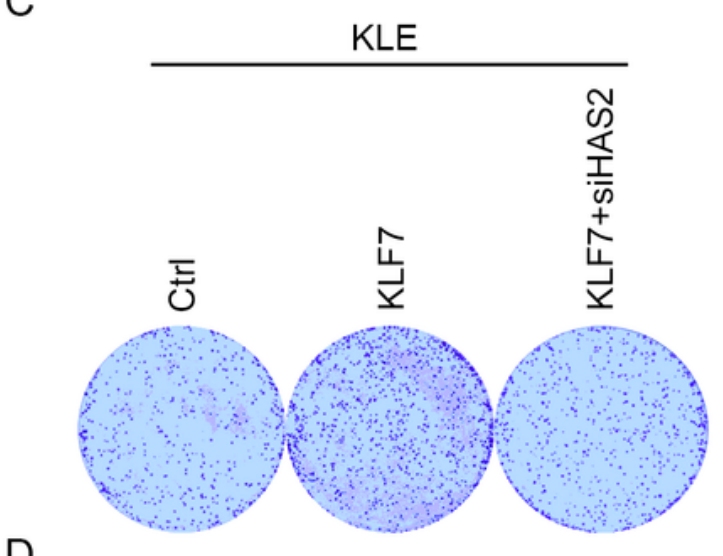

D

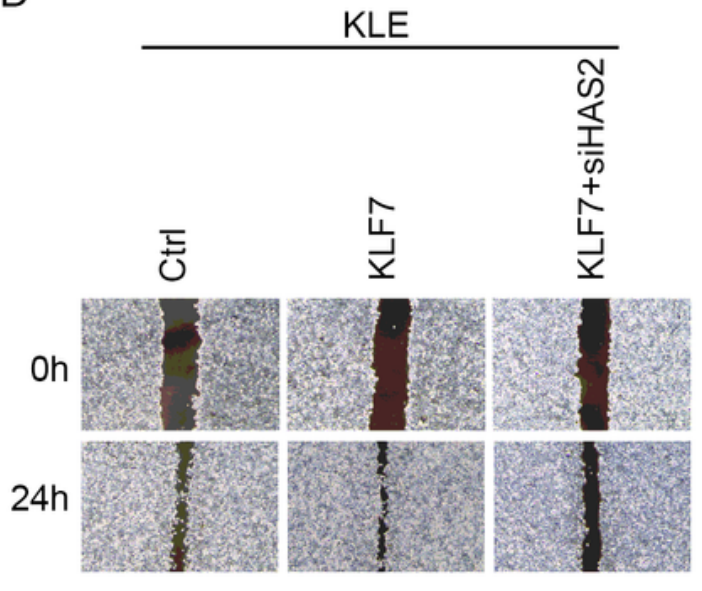

B

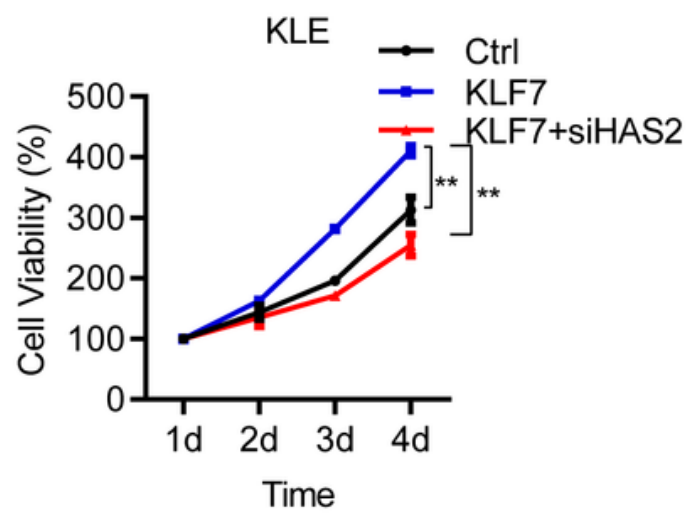

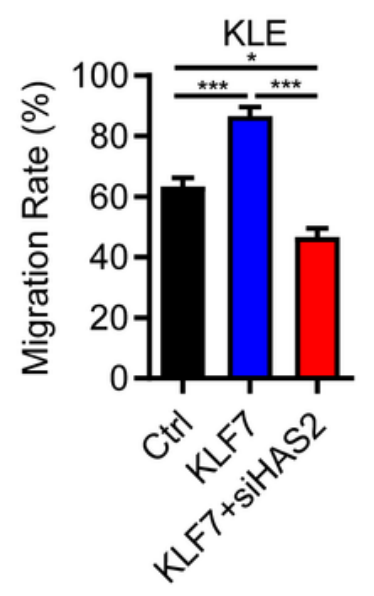

\section{Figure 7}

KLF7 upregulation of HAS2 promotes UCEC cell proliferation and migration (A) Immunoblotting analysis of HAS2 in Ctrl, KLF7 and KLF7+siHAS2 KLE cells. (B) CCK8 analysis of proliferation in Ctrl, KLF7 and KLF7+siHAS2 KLE cells. (C) Colony formation was assessed in Ctrl, KLF7 and KLF7+siHAS2 KLE cells. (D) Migration was assessed by wound healing in Ctrl, KLF7 and KLF7+siHAS2 KLE cells. ${ }^{*} p<0.05$. ${ }^{* *} \mathrm{p}<0.01 .{ }^{* * *} \mathrm{p}<0.001$. 


\section{Supplementary Files}

This is a list of supplementary files associated with this preprint. Click to download.

- ARRIVEGuidelinesChecklist.docx

- SupplementaryFile.docx 